\title{
ANALYSIS OF VIBRATION TRANSFER CHARACTERISTICS OF VEHICLE SUSPENSION SYSTEM EMPLOYING INERTER
}

\author{
Yujie Shen, Long Chen, Yanling Liu, Xiaoliang Zhang \\ School of Automotive and Traffic Engineering, Jiangsu University, Zhenjiang, China \\ e-mail: shenliang6018@163.com; chenlong@ujs.edu.cn; liuyl@ujs.edu.cn; zxl1979@ujs.edu.cn
}

In this paper, the force transfer mechanism of three mechanical elements "inerter, spring and damper" is analyzed based on the "force-current" analogy theory. The vibration isolation performance of the two types of simple three-element vehicle suspensions S1 (inerter is in parallel with damper) and S2 (inerter is in series with damper) are studied. The dual-mass system model of the suspensions is built by means of using the mechanical impedance method. The influence of parameters variation on vibration transfer characteristics is also investigated.

Keywords: vehicle, suspension, inerter, vibration analysis

\section{Introduction}

The vehicle suspension is a general term for power transmission device located between wheels and axles. The performance of suspension has a great impact on the vehicle ride comfort, handle and stability (Rajamani, 2012). There are three types of vehicle suspensions including passive suspension, semi-active (Eltantawie, 2012; Huang et al., 2013; Tudon-Martinez et al., 2013) and active suspension (Hac, 1992; Roh and Park, 1999; Youn and Hac, 2006). Recently, a new type of vehicle suspension employing an inerter has drawn the attention of scholars.

The inerter (Smith, 2002) is a newly proposed two-terminal element. It has a characteristic that the force applying to the two terminals is proportional to relative acceleration. The dynamic equation is

$$
F=b\left(\dot{v}_{1}-\dot{v}_{2}\right)
$$

where $F$ is the force applying to the two terminals, $v_{1}, v_{2}$ are velocities of the two terminals, $b$ is the inertance, the unit is $\mathrm{kg}$.

In the "force-current" analogy between mechanical circuit and electric circuit, the mass, spring and damper correspond to capacitor, inductor and resistor. There is a restriction in the mass element for its one-terminal property, so that the capacitor should be grounded. With the birth of the inerter, the mechanical circuit mass element is replaced by the inerter corresponding to the capacitor in an electric circuit. Also, the traditional mechanical spring-damper network performance can be improved by inclusion of the inerter and has been widely used in vehicle suspensions (Smith and Wang, 2004; Papageorgiou and Smith, 2006; Kuznetsov et al., 2011; Hu et al., 2014; Wang and Chan, 2011), train suspensions (Wang et al., 2009; Wang and Liao, 2010), building suspensions (Wang et al., 2010) and the steering compensation for high-performance motorcycles (Evangelou et al., 2004).

The earliest types of inerters are rack-and-pinion and ball-screw inerters (Papageorgiou and Smith, 2005). Experiments were also carried out to verify the effectiveness of mechanical inerters. Furthermore, the influence of the inerter on natural frequencies of vibration systems 
was investigated (Chen et al., 2014) to demonstrate that the inerter can reduce the natural frequencies of the vibration system. The benefits of semi-active suspensions with inerters were investigated by Zhang and Mehdi (2012). It was also observed that the inerter and spring force were in anti-phase to each other (Ming et al., 2014), but the force transfer mechanism of the three elements and the influence of the parameters on the vibration transfer characteristics of the vehicle suspension remained unknown.

This paper intends to study the force transfer mechanism of the inerter, spring and damper based on the "force-current" analogy, and to investigate the effectiveness of the mechanical network compromising the inerter. Meanwhile, vibration transfer characteristics of the vehicle suspension are also studied to draw some conclusions. The paper is arranged as follows:

In Section 2, the force transfer mechanism of the three types of elements is analyzed. Then, a single mass model is built in Section 3 to demonstrate the effectiveness of the new mechanical network compromising the inerter from the perspective of the force transferred in suspension. In order to analyze the influence of variable parameters on the vibration transfer characteristics, a dual mass model is built in Section 4, and the effect is studied in Section 5. At last, some conclusions are drawn in Section 6.

\section{Force transfer mechanism of the three elements}

The force and velocity in a mechanical field correspond to the current and voltage in an electric field. The force and current are both "through-variables" while velocity and voltage are both "across-variables". In the current transfer process, it is known that the current phase is ahead of the voltage phase between two terminals of the capacitor. But for the inductor, the voltage phase is ahead of the current phase. In the mechanical element, the direction of the force between two terminals of an inerter is always opposite to that of the spring (Ming et al., 2014). In the following, the force transfer mechanism of the three elements will be studied. Figure 1 shows the force transfer model of the three elements, where $q_{0}$ is displacement of the input, $k_{0}$ is stiffness, $b_{0}$ is inertance, $c_{0}$ is damping coefficient.

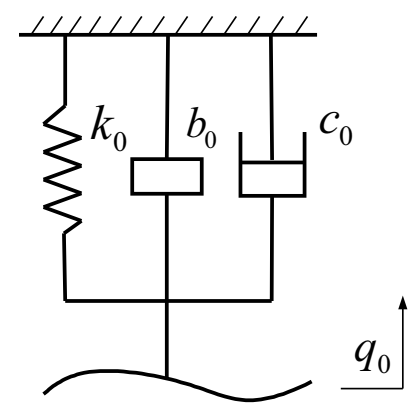

Fig. 1. Force transfer model

The force between the two terminals of the spring, inerter and damper $f_{k}, f_{b}$, and $f_{c}$ are

$$
f_{k}=k_{0} q_{0} \quad f_{b}=b_{0} \ddot{q}_{0} \quad f_{c}=c_{0} \dot{q}_{0}
$$

when $q_{0}$ is set as

$$
q_{0}=A \sin (w t)
$$

where $A$ is the amplitude equal to $0.1 \mathrm{~m}$ and $w$ is the circle frequency set as $2 \pi$. The velocity between the two terminals is

$$
\dot{q}_{0}=A w \cos (w t)
$$


In simulation, $k_{0}=10 \mathrm{~N} / \mathrm{m}, c_{0}=1.5 \mathrm{~N} \mathrm{~s} / \mathrm{m}, b_{0}=0.1 \mathrm{~kg}$.

Figures $2 \mathrm{a}$ to $2 \mathrm{c}$ show the force and velocity of the two terminals of the spring, inerter, and damper.
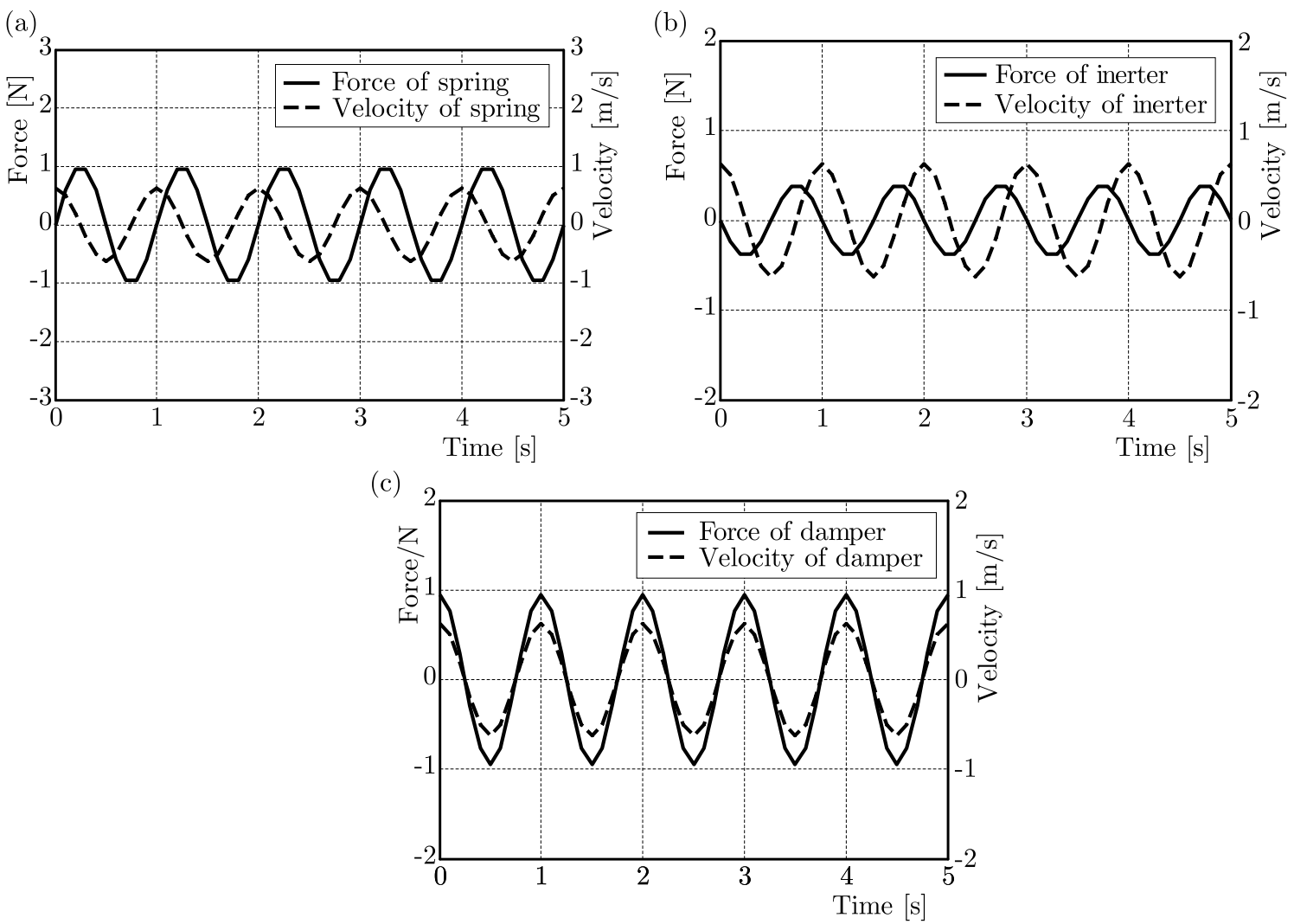

Fig. 2. Force and velocity of the two terminals of (a) the spring, (b) the inerter, (c) the damper

It can be seen that the velocity phase of the spring is ahead of the force phase, which is consistent with the relationships between the voltage and current phase of the inductor. For inerter, the force phase is ahead of the velocity phase which is consistent with the relationships between the current phase and the voltage phase of the capacitor. The force phase is always synchronous with the velocity phase, which is consistent with the relationships between the current phase and the voltage phase of the resistor. The analysis above demonstrates the effectiveness of the "force-current" analogy again.

\section{Single mass system model}

There are many types of vehicle suspensions employing the inerter. In this paper, two types of a simple three-element vehicle suspension are investigated. Both of them have a spring to bear the sprung mass. For S1 suspension, the inerter is in parallel with the damper. For S2 suspension, the inerter is in series with the damper. Two types of the suspension structures and the traditional passive suspension S0 compromising parallel "spring and damper" are shown in Fig. $3\left(z, z_{1}, z_{2}\right.$ are displacements of the mass, $q, q_{1}, q_{2}$ are displacements of the input, $z_{b}$ is displacement of the inerter, $k, k_{1}, k_{2}$ are stiffnesses of springs, $c, c_{1}, c_{2}$ are damping coefficients, $b_{1}, b_{2}$ are inertances).

The force in $\mathrm{S} 0$ is

$$
f_{S_{0}}=f_{k}+f_{c}=k(q-z)+c(\dot{q}-\dot{z})
$$




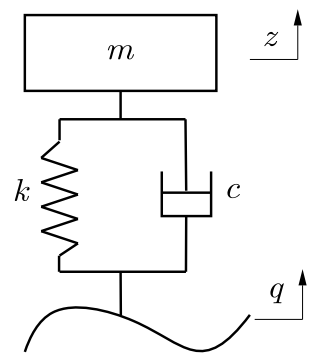

S0

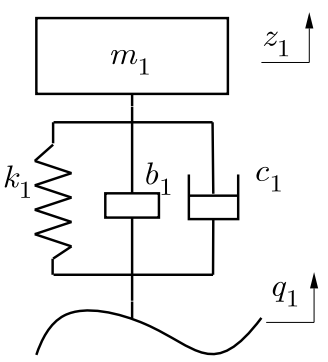

S1

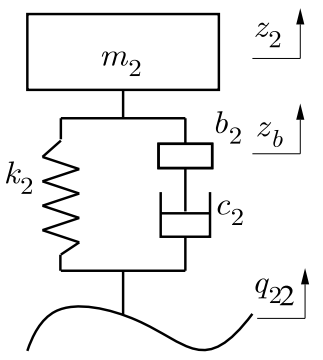

S2

Fig. 3. Three types of the suspension structure

The force in $\mathrm{S} 1$ is

$$
f_{S_{1}}=f_{k}+f_{b}+f_{c}=k_{1}\left(q_{1}-z_{1}\right)+b_{1}\left(\ddot{q}_{1}-\ddot{z}_{1}\right)+c\left(\dot{q}_{1}-\dot{z}_{1}\right)
$$

The force in $\mathrm{S} 2$ is

$$
f_{S_{2}}=f_{k}+b_{2}\left(\ddot{z}_{b}-\ddot{z}_{2}\right)
$$

where

$$
b_{2}\left(\ddot{z}_{b}-\ddot{z}_{2}\right)=c_{2}\left(\dot{q}_{2}-\dot{z}_{b}\right)
$$

Assuming the $\lambda$ is

$$
\lambda=\frac{w}{w_{0}} \quad w_{0}=\sqrt{\frac{k}{m}}
$$

The model parameters are all kept the same, see Table 1.

Table 1. Model parameters

\begin{tabular}{|l|c|}
\hline \multicolumn{1}{|c|}{ Parameters } & Value \\
\hline \hline Sprung mass $m, m_{1}, m_{2}[\mathrm{~kg}]$ & 320 \\
\hline Stiffness $k, k_{1}, k_{2}[\mathrm{k} \mathrm{N} / \mathrm{m}]$ & 15 \\
\hline Inertance $b_{1}, b_{2}[\mathrm{~kg}]$ & 10 \\
\hline Damping coefficient $c, c_{1}, c_{2}[\mathrm{~N} \mathrm{~s} / \mathrm{m}]$ & 1095 \\
\hline
\end{tabular}

The input displacement is also set as a sine wave with an amplitude of $0.1 \mathrm{~m}$. Figure 4 shows the amplitude ratio of the force in S1 and S2 with S0.

The solid line corresponds to the constant value of 1 . When the amplitude of the ratio of the force in the suspension structure is less than 1, it indicates that the vibration isolation performance is superior to the traditional passive suspension. In the range from 0 to 8 of $\lambda$, both ratios S1 and S2 are less than 1, which means that their vibration isolation performance is superior to the traditional passive suspension in low frequency. The amplitude of ratio of S1 is becoming larger than 1 with an increase in $\lambda$ but for S2, the amplitude of the ratio is always less than 1 . It can be inferred that the vibration isolation performance of the mechanical circuit is improved in the low frequency by involving of the inerter element, which is consistent with the capacitor function to block the low frequency current. Also, suspension S2 has a superior isolation performance with respect to $\mathrm{S} 1$ and is more suitable for vehicle suspension design. 


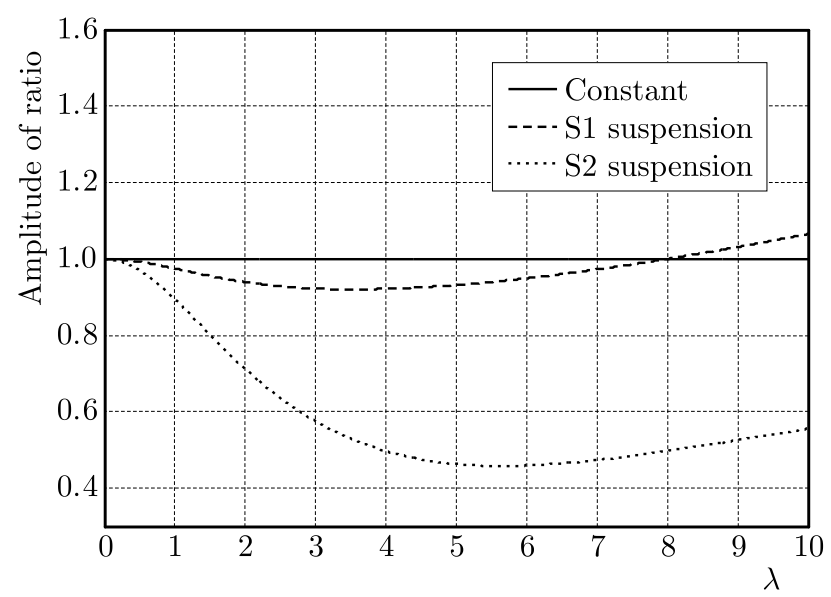

Fig. 4. Amplitude ratio of the force

\section{Dual-mass system model}

In the "force-current" analogy, the spring, damper and inerter have the same forms of dynamic equations like the inductor, resistor and capacitor. So, the impedances of the spring, damper and inerter also have the same forms of $k / s, c$ and $b s$, just like the electric impedance $1 /(L s), C s$ and $1 / R$ of inductor, resistor and capacitor, where $k$ is stiffness of the spring, $c$ is the damping coefficient, $b$ is the inertance, $L$ is the inductance coefficient, $C$ is the capacitance coefficient, $R$ is the resistance coefficient. Analysis of vibration transfer characteristics in the frequency domain can become more convenient by using the impedance methods. A dual mass system model compromising the sprung and unsprung mass is built in Fig. 5, where $m_{s}$ is the sprung mass, $m_{u}$ is the unsprung mass, $k_{t}$ is the stiffness of the tire, $z_{s}$ is the displacement of the sprung mass, $z_{u}$ is the displacement of the unsprung mass, $z_{r}$ is the displacement of the road input, $T(s)$ is the velocity impedance of the suspension.

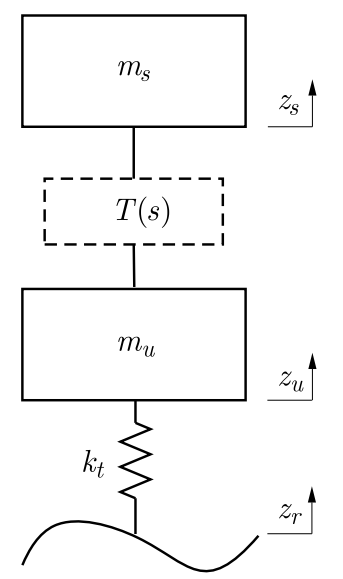

Fig. 5. Dual mass system

The dynamic model is

$$
\begin{aligned}
& m_{s} s^{2} Z_{s}+s T(s)\left(Z_{s}-Z_{u}\right)=0 \\
& m_{u} s^{2} Z_{u}-s T(s)\left(Z_{s}-Z_{u}\right)+k_{t}\left(Z_{u}-Z_{r}\right)=0
\end{aligned}
$$

where $Z_{s}, Z_{u}$ and $Z_{r}$ are the Laplace transforms of $z_{s}, z_{u}$ and $z_{r}$ and: 
- for S1

$$
T_{1}(s)=\frac{k_{1}}{s}+b_{1} s+c_{1}
$$

- for S2

$$
T_{2}(s)=\frac{k_{2}}{s}+\frac{1}{\frac{1}{b_{2} s}+\frac{1}{c_{2}}}
$$

So, the gain of the body acceleration is

$$
H(s)_{\ddot{z}_{s} \sim z_{r}}=\frac{Z_{s}}{Z_{r}} s^{2}=\frac{T(s) k_{t} s^{2}}{\left[m_{s} s+T(s)\right]\left[m_{u} s^{2}+s T(s)+k_{t}\right]-s T^{2}(s)}
$$

The gain of the suspension deflection is

$$
H(s)_{\left(z_{s}-z_{u}\right) \sim z_{r}}=\frac{Z_{s}-Z_{u}}{Z_{r}}=\frac{-m_{s} s k_{t}}{\left[m_{s} s+T(s)\right]\left[m_{u} s^{2}+s T(s)+k_{t}\right]-s T^{2}(s)}
$$

The gain of the dynamic tire load is

$$
H(s)_{\left(z_{u}-z_{r}\right) k_{t} \sim z_{r}}=\frac{Z_{u}-Z_{r}}{Z_{r}} k_{t}=\left(\frac{\left[m_{s} s+T(s)\right] k_{t}}{\left[m_{s} s+T(s)\right]\left[m_{u} s^{2}+s T(s)+k_{t}\right]-s T^{2}(s)}-1\right) k_{t}
$$

In simulation, the parameters of the suspensions are optimized by using a genetic algorithm. The model parameters are shown in Table 2. In order to show the performance of the vehicle suspension employing the inerter, a traditional passive suspension (stiffness is $22 \mathrm{kN} / \mathrm{m}$ and damping coefficient is $1000 \mathrm{~N} \mathrm{~s} / \mathrm{m}$ ) is set as a comparison object.

Table 2. Simulation parameters

\begin{tabular}{|l|c|}
\hline \multicolumn{1}{|c|}{ Parameters } & Values \\
\hline \hline Sprung mass $m_{s}[\mathrm{~kg}]$ & 320 \\
\hline Unprung mass $m_{u}[\mathrm{~kg}]$ & 45 \\
\hline Stiffness of tire $k_{t}[\mathrm{kN} / \mathrm{m}]$ & 190 \\
\hline Stiffness of S1 $k_{1}[\mathrm{kN} / \mathrm{m}]$ & 28 \\
\hline Damping coefficient of S1 $c_{1}[\mathrm{~N} \mathrm{~s} / \mathrm{m}]$ & 2800 \\
\hline Inertance of S1 $b_{1}[\mathrm{~kg}]$ & 20 \\
\hline Stiffness of S2 $k_{2}[\mathrm{kN} / \mathrm{m}]$ & 22 \\
\hline Damping coefficient of S1 $c_{2}[\mathrm{~N} \mathrm{~s} / \mathrm{m}]$ & 1300 \\
\hline Inertance of S2 $b_{2}[\mathrm{~kg}]$ & 500 \\
\hline
\end{tabular}

The other parameters are remained unchanged in order to analyze the effect of one parameter. The variation of the parameters are shown in Table 3.

Table 3. Parameters

\begin{tabular}{|c|c|c|c|c|c|c|}
\hline & $\begin{array}{c}b_{1} \\
{[\mathrm{~kg}]}\end{array}$ & $\begin{array}{c}b_{2} \\
{[\mathrm{~kg}]}\end{array}$ & $\begin{array}{c}c_{1} \\
{[\mathrm{kN} \mathrm{s} / \mathrm{m}]}\end{array}$ & $\begin{array}{c}c_{2} \\
{[\mathrm{kN} \mathrm{s} / \mathrm{m}]}\end{array}$ & $\begin{array}{c}k_{1} \\
{[\mathrm{kN} / \mathrm{m}]}\end{array}$ & $\begin{array}{c}k_{2} \\
{[\mathrm{kN} / \mathrm{m}]}\end{array}$ \\
\hline \hline Value & 20 & 500 & 2.8 & 1.3 & 28 & 22 \\
\hline$+6 \mathrm{~dB}$ & 40 & 1000 & 5.6 & 2.6 & 56 & 44 \\
\hline$-6 \mathrm{~dB}$ & 10 & 250 & 1.4 & 0.65 & 14 & 11 \\
\hline
\end{tabular}




\section{Analysis of vibration transfer characteristics}

\subsection{Effect of variable stiffness}

Firstly, the damping coefficient and the inertance remain unchanged. Figures 6-8 show the gains of body acceleration, suspension deflection and dynamic tire load of variable stiffness. The red line represents passive suspension S0 while the blue and green lines represent S1 and S2 suspensions. The direction of the arrows means an increase of the stiffness.

In Fig. 6, it can be seen that both the gains of the body acceleration of S1 and S2 become larger and even beyond S0 with an increase of the stiffness in low frequency. But in high frequency, the gains become smaller and slightly lower than S0 in S1 while become larger and higher than S0 in S2. Furthermore, both their resonance frequencies become larger with an increase of stiffness.
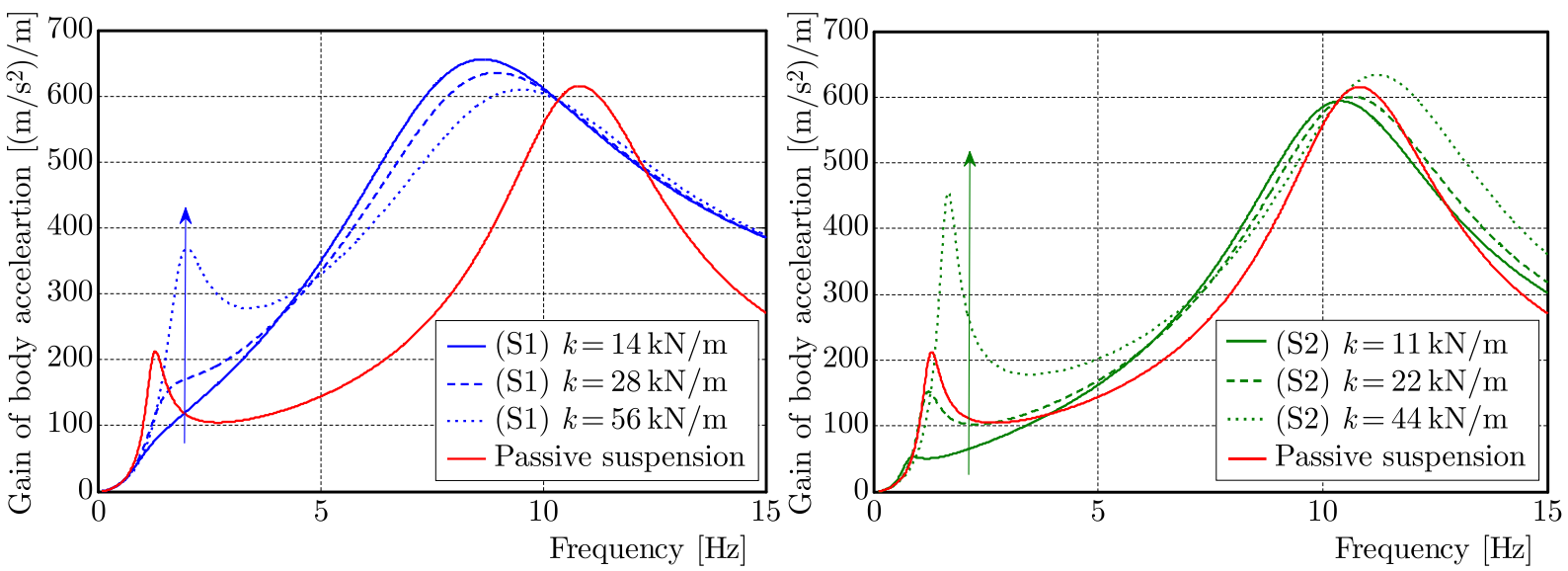

Fig. 6. Gains of body acceleration of variable stiffness

Figure 7 shows the gains of suspension deflection of variable stiffness. Both their gains and resonance frequencies become larger with an increase of stiffness except for the gains in high frequency. Note that all the peak values of S1 and S2 are smaller than S0 except for S2 where $k=44 \mathrm{kN} / \mathrm{m}$. At last, the gains of dynamic tire load of variable stiffness are shown in Fig. 8.
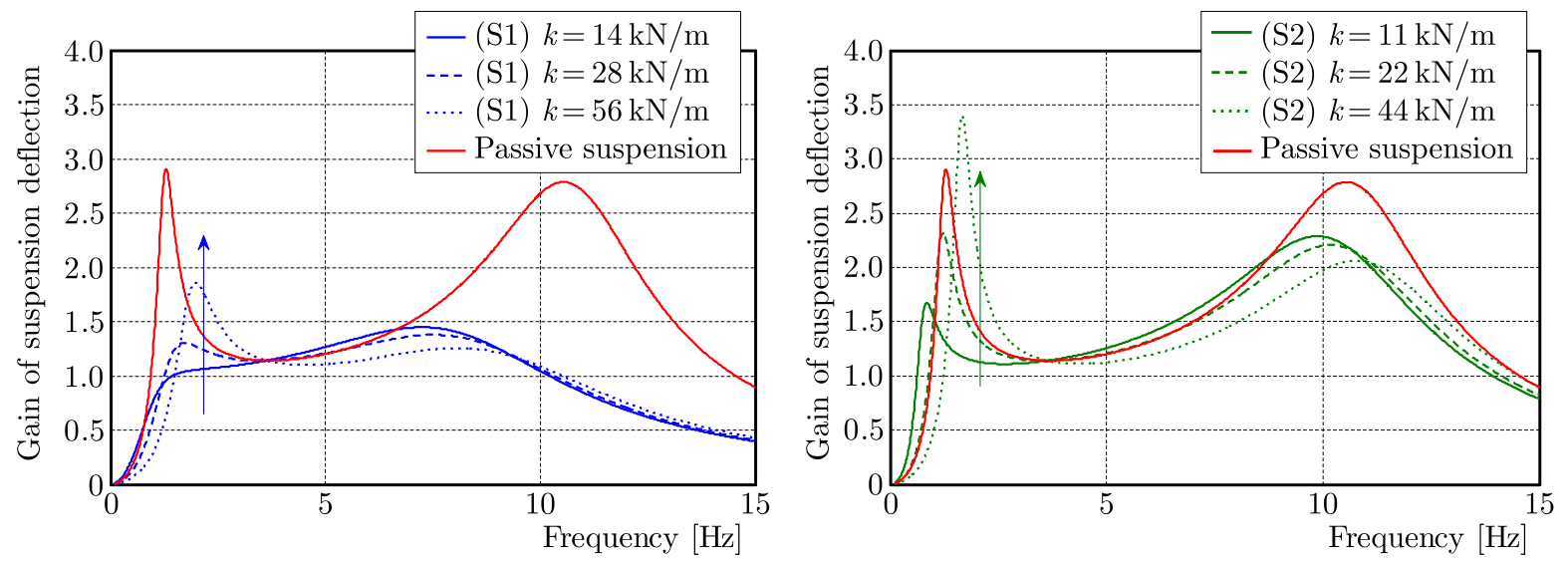

Fig. 7. Gains of suspension deflection of variable stiffness

It can be seen that the trends are all consistent with the suspension deflection that their gains become larger in low frequency and become smaller in high frequency. Compared with the passive suspension, the peak value in low frequency becomes larger than S0 but always smaller 

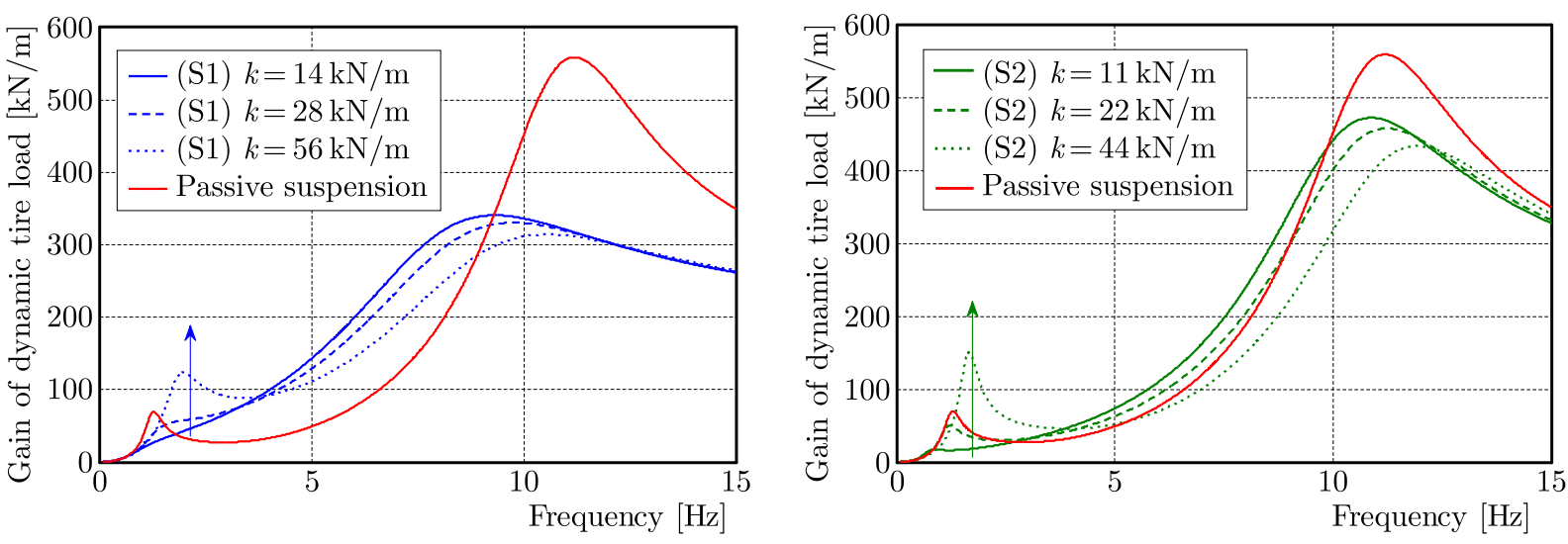

Fig. 8. Gains of dynamic tire load of variable stiffness

than S0 in high frequency. Both the resonance frequencies become larger with an increase of stiffness.

It can be concluded that the gains of S1 and S2 become larger in low frequency and become smaller in high frequency except for the gains of body acceleration of S2 with an increase of stiffness. Both their resonance frequencies become larger with an increase of stiffness.

\subsection{Effect of variable damping coefficient}

Then, the stiffness and the inertance remain unchanged. Figures 9-11 show the gains of body acceleration, suspension deflection and dynamic tire load of variable damping coefficient. The red line represents passive suspension S0 while the blue and green lines represent S1 and S2 suspensions. The direction of the arrows means an increase of the damping coefficient.

It can be seen that both the gains of the body acceleration become smaller than S0 at the original two resonance frequencies with an increase of the damping coefficient. Furthermore, the original two resonances change to one resonance.
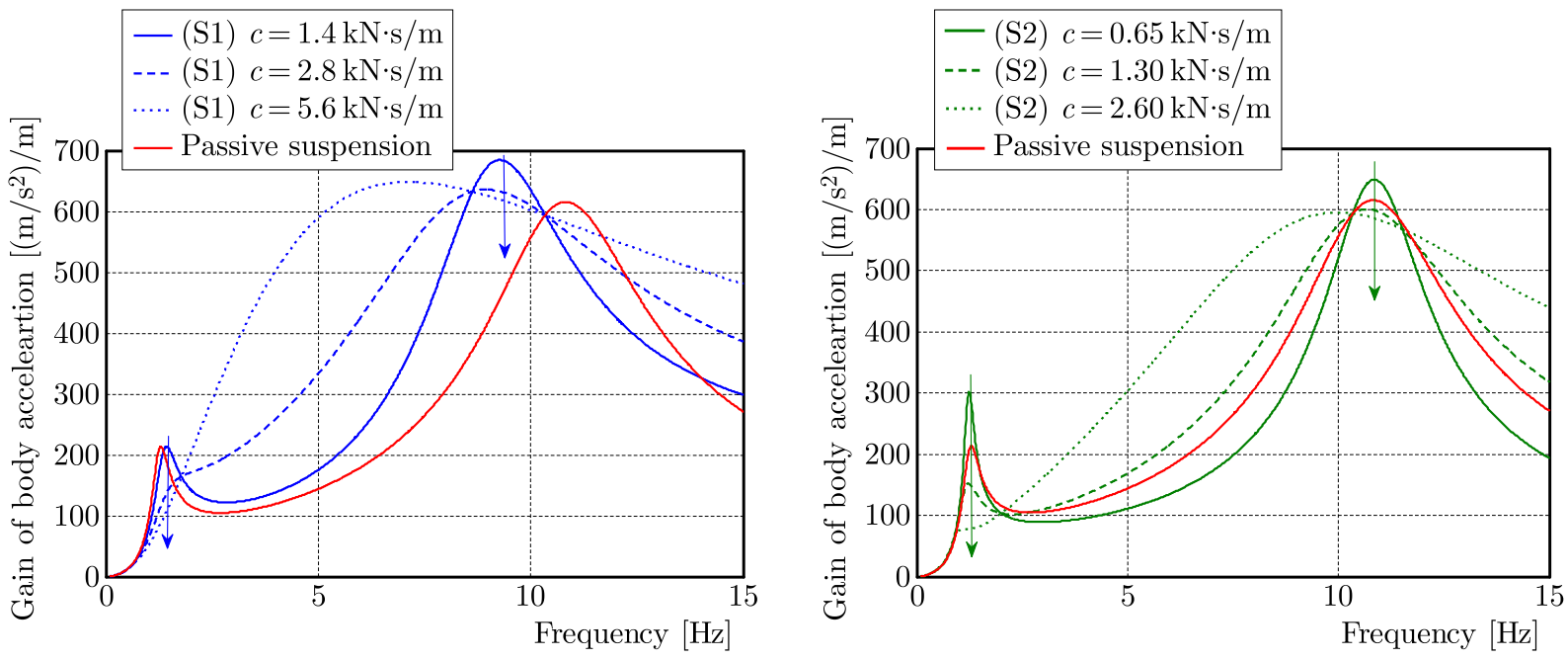

Fig. 9. Gains of body acceleration of variable damping coefficient

Figure 10 shows the gains of suspension deflection of the variable damping coefficient. The trends are consistent with the body acceleration that their gains become smaller than S0 at the original two resonance frequencies with an increase of the damping coefficient. At the same time, the original two resonances change to one resonance. At last, the gains of dynamic tire load of the variable damping coefficient are shown in Fig. 11. 

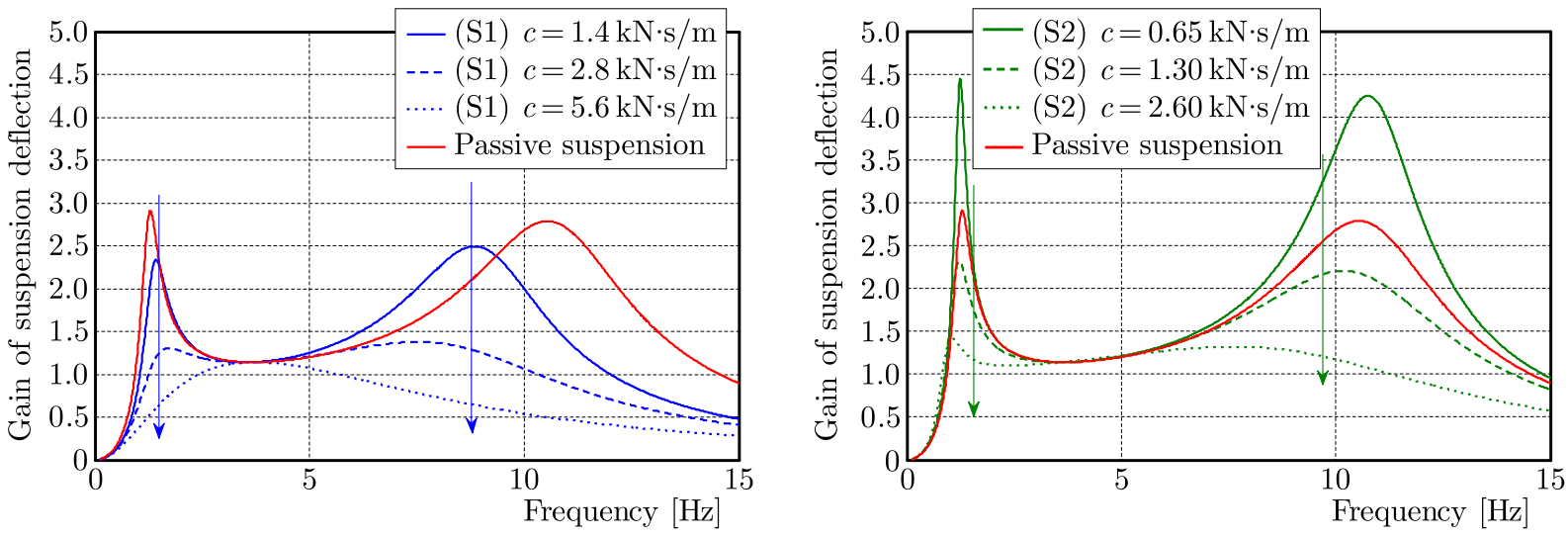

Fig. 10. Gains of suspension deflection of variable damping coefficient
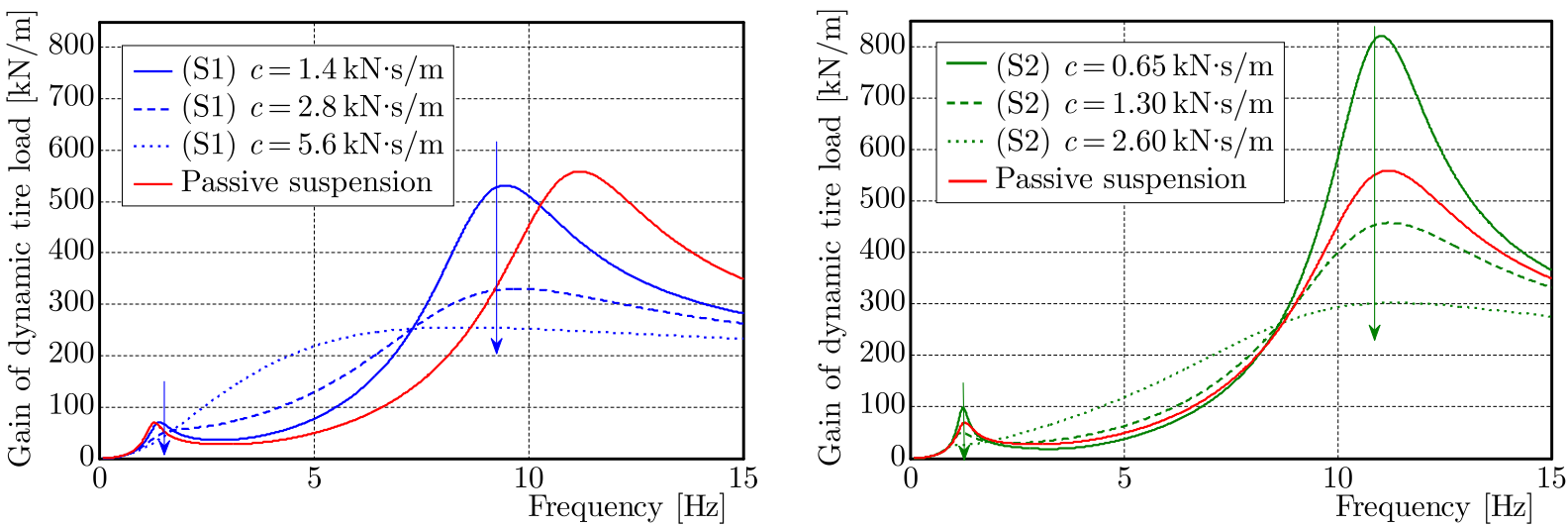

Fig. 11. Gains of dynamic tire load of variable damping coefficient

It can be seen that the trends are all consistent with the suspension deflection and body acceleration that their gains become smaller than $\mathrm{S} 0$ at the original two resonance frequencies, The original two resonances change to one resonance with an increase of the damping coefficient.

It can be concluded that the gains of S1 and S2 have the same trends that their gains become smaller than $\mathrm{S} 0$ at the original two resonance frequencies, and the original two resonances change to one resonance with an increase of the damping coefficient.

\subsection{Effect with variable inertance}

Lastly, the stiffness and the damping coefficient remain unchanged. Figures 12-14 show the gains of body acceleration, suspension deflection and dynamic tire load of variable inertance. The red line represents passive suspension S0 while the blue and green lines represent S1 and S2 suspensions. The direction of the arrows means an increase of the inertance.

It can be seen that both the gains of the body acceleration of S1 and S2 become smaller than S0 in low frequency, and the resonance frequencies become smaller in S1 while become larger in S2 with an increase of inertance. But in high frequency, the gains of S1 become larger than S0 and the resonance frequencies become smaller while there is no obvious change in S2.

Figure 13 shows the gains of suspension deflection of variable inertance. The trends are consistent with the body acceleration that both the gains become smaller than $\mathrm{S} 0$ in low frequency and the resonance frequencies become smaller in S1 while become larger in S2 with an increase of the inertance. But in high frequency, the gains of S1 become larger but still less than S0, and the resonance frequencies become smaller while there is no obvious change in S2. At last, the gains of dynamic tire load of variable inertance are shown in Fig. 14. 

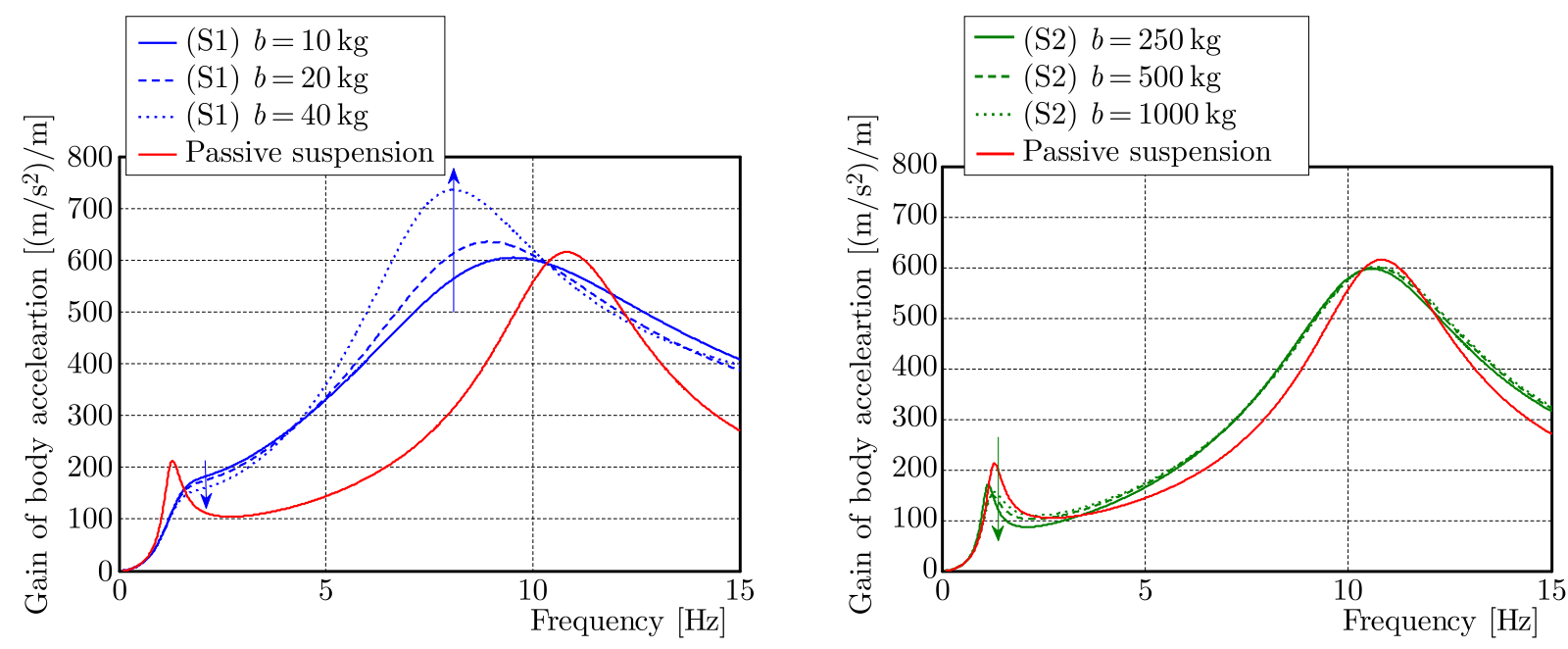

Fig. 12. Gains of body acceleration of variable inertance
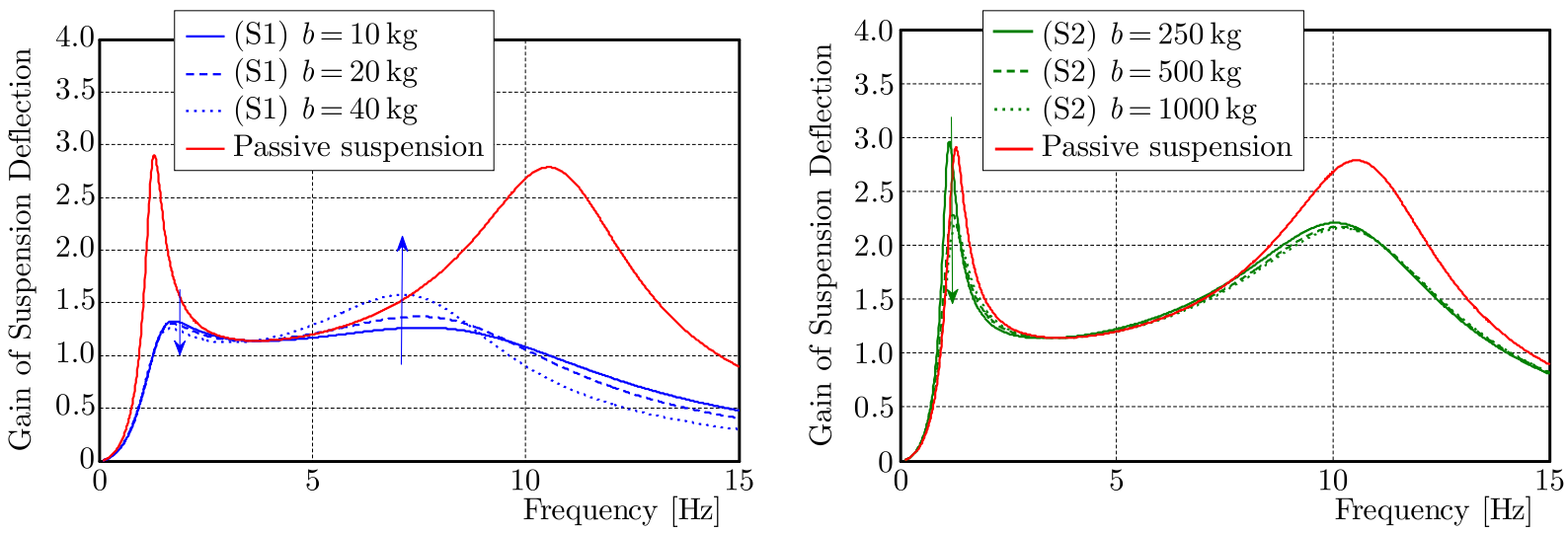

Fig. 13. Gains of suspension deflection of variable inertance
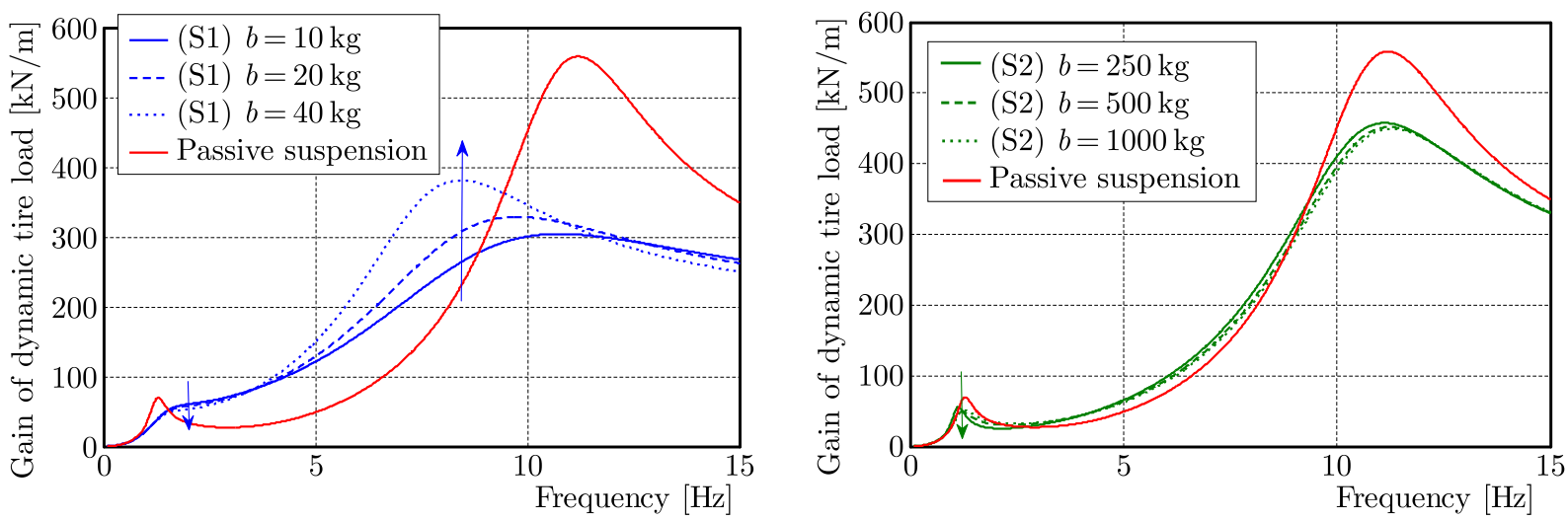

Fig. 14. Gains of dynamic tire load of variable inertance

It can be seen that the trends are all consistent with the suspension deflection and body acceleration that both of the gains become smaller than S0 in low frequency and the resonance frequencies become smaller slightly in S1 while become larger slightly in S2 with an increase of inertance. But in high frequency, the gains of S1 become larger but still less than S0, and the resonance frequencies become smaller while there is no obvious change in S2.

It can be concluded that the gains of S1 and S2 have the same trends that both the gains become smaller than $\mathrm{S} 0$ in low frequency and the resonance frequencies become smaller in $\mathrm{S} 1$ 
while become larger in S2 with an increase of inertance. But in high frequency, the gains of S1 become larger but still less than S0 except for the body acceleration where $b=20 \mathrm{~kg}$ and $40 \mathrm{~kg}$, and the resonance frequencies become smaller while there is no obvious change in S2.

\section{Conclusion}

In this paper, the force transfer mechanism of the three types of mechanical elements, i.e. the inerter, spring and damper are analyzed. A "force-current" analogy is further demonstrated that the force phase is ahead of the velocity phase in the inerter, the velocity phase is ahead of the force phase in the spring, while the force phase is always synchronous with the velocity phase in the damper. Then, the vibration isolation performance of S1 and S2 suspensions are deemed improved by compromising the inerter in low frequency from the prospective of the force transferred. At last, the dual-mass model of the suspension is built by means of the impedance method. The velocity impedances of the mechanical elements are used in frequency analysis for they have the same forms of dynamic equations of the electric elements according to the "forcecurrent" analogy. The influences on the vibration transfer characteristics of the parameters variation are investigated. The conclusions are drawn that the gains of S1 and S2 become larger in low frequency and become smaller in high frequency, except for the gain of body acceleration in S2 with an increase of stiffness. Both their resonance frequencies become larger with an increase of stiffness. For the damping coefficient, the gains of S1 and S2 have the same trends that their gains become smaller at the original two resonance frequencies, and the original two resonances change to one resonance with an increase of the damping coefficient. With an increase of inertance, the gains of S1 and S2 have the same trend that both the gains become smaller in low frequency and the resonance frequencies becomes smaller in S1 while become larger in S2. But in high frequency, the gains of $\mathrm{S} 1$ become larger and the resonance frequencies become smaller while there is no obvious change in S2.

\section{Acknowledgement}

This work is supported by the National Natural Science Foundation of China (Grant No. 51405202), Scientific Research Innovation Projects of Jiangsu Province (Grant No. KYLX15_1081), China Postdoctoral Science Foundation (Grant No. 2014M561591), the "Six Talent Peaks" high-level project of Jiangsu Province (Grant No. 2014-JNHB-023) Yujie Shen is also supported by the China Scholarship Council.

\section{References}

1. Chen M.Z.Q., Hu Y.L., Huang L.X., 2014, Influence of inerter on natural frequencies of vibration systems, Journal of Sound and Vibration, 333, 7, 1874-1887

2. Eltantawie M.A., 2012, Decentralized neuro-fuzzy control for half car with semi-active suspension system, International Journal of Automotive Technology, 13, 3, 423-431

3. Evangelou S., Limebeer D.J.N., Sharp R.S., 2004, Steering compensation for high performance motorcycles, Proceedings of the 43rd IEEE Conference on Design and Control, 749-754

4. HAC A., 1992, Optimal linear preview control of active vehicle suspension, Vehicle System Dynamics, 21, 1, 167-195

5. Huang C., Chen L., Yuan C.C., Jiang H.B., 2013, Non-linear modelling and control of semiactive suspensions with variable damping, Vehicle System Dynamics, 51, 10, 1568-1587

6. Hu Y.L., Chen M.Z.Q., Shu Z., 2014, Passive vehicle suspensions employing inerters with multiple performance requirements, Journal of Sound and Vibration, 333, 8, 2212-2225 
7. Kuznetsov A., Mammadov M., Sultan I., 2011, Optimization of improved suspension system with inerter device of the quarter-car model in vibration analysis, Archive of Applied Mechanics, 81, 10, 1427-1437

8. Ming F.S., Rahizar R., Wan N.L.W.M., 2014, Vehicle suspension with parallel inerter: effectiveness in improving vibration isolation, Journal of Vibroengineering, 16, 1, 255-265

9. Papageorgiou C., Smith M.C., 2005, Laboratory experimental testing of inerters, Proceedings of the 44th IEEE Conference on Decision and Control, and the European Control Conference, 3351-3356

10. Papageorgiou C., Smith M.C., 2006, Positive real synthesis using matrix inequalities for mechanical networks: application to vehicle suspension, IEEE Transactions on Control System Technology, 14, 3, 423-434

11. Rajamani R., 2012, Vehicle Dynamics and Control, Springer, New York

12. RoH H., PARK Y., 1999, Stochastic optimal preview control of an active vehicle suspension, Journal of Sound and Vibration, 220, 2, 313-330

13. Smith M.C., Wang F.C., 2004, Performance benefits in passive vehicle suspensions employing inerters, Vehicle System Dynamics, 42, 4, 235-257

14. Sмith M.C., 2002, Synthesis of mechanical networks: the inerter, IEEE Transactions on Automatic Control, 47, 10, 1648-1662

15. Tudon-Martinez J., Fergani S., Verrier S., Sename O., Dugard L., MoralesMenendez R., Ramirez-MendozA R.A., 2013, Road adaptive semi-active suspension in an automotive vehicle using an LPV controller, Advances in Automotive Control, 7, 231-236

16. Wang F.C., Chan H., 2011, Vehicle suspension with a mechatronic network strut, Vehicle System Dynamics, 49, 5, 811-830

17. Wang F.C., Hong M.F., Chen C.W., 2010, Building suspension with inerters, Proceedings of the Institution of Mechanical Engineers, Part C: Journal of Mechanical Engineering Science, 224, 8, 1605-1616

18. WAng F.C., Liao M. K., 2010, The lateral stability of train suspension systems employing inerters, Vehicle System Dynamics, 48, 5, 619-643

19. Wang F.C., Liao M.K., Liao B.H., 2009, The performance improvements of train suspension systems with mechanical networks, Vehicle System Dynamics, 47, 7, 805-830

20. Youn I., Hac A., 2006, Preview control of active suspension with integral action, International Journal of Automotive Technology, 7, 5, 547-554

21. Zhang X.J., Mehdi A., 2012, On the benefits of semi-active suspensions with inerters, Shock and Vibration, 19, 257-272 